\title{
Multi-point boundary value problems for a coupled system of nonlinear fractional differential equations
}

\author{
Chengbo Zhai* and Mengru Hao
}

\section{"Correspondence:}

cbzhai@sxu.edu.cn

School of Mathematical Sciences,

Shanxi University, Taiyuan, Shanxi

030006, P.R. China

\section{Springer}

\begin{abstract}
In this paper, we investigate the existence and uniqueness of solutions to the coupled system of nonlinear fractional differential equations

$$
\left\{\begin{array}{l}
-D_{0^{+}}^{\nu_{1}} y_{1}(t)=\lambda_{1} a_{1}(t) f\left(y_{1}(t), y_{2}(t)\right) \\
-D_{0^{+}}^{\nu_{2}} y_{2}(t)=\lambda_{2} a_{2}(t) g\left(y_{1}(t), y_{2}(t)\right)
\end{array}\right.
$$

where $D_{0^{+}}^{v}$ is the standard Riemann-Liouville fractional derivative of order $\nu, t \in(0,1)$, $\boldsymbol{v}_{1}, \boldsymbol{v}_{2} \in(n-1, n]$ for $n>3$ and $n \in \mathbf{N}$, and $\lambda_{1}, \lambda_{2}>0$, with the multi-point boundary value conditions: $y_{1}^{(i)}(0)=0=y_{2}^{(i)}(0), 0 \leq i \leq n-2 ; D_{0^{+}}^{\beta} y_{1}(1)=\sum_{i=1}^{m-2} b_{i} D_{0^{+}}^{\beta} y_{1}\left(\xi_{i}\right)$; $D_{0^{+}}^{\beta} y_{2}(1)=\sum_{i=1}^{m-2} b_{i} D_{0^{+}}^{\beta} y_{2}\left(\xi_{i}\right)$, where $n-2<\beta<n-1,0<\xi_{1}<\xi_{2}<\cdots<\xi_{m-2}<1$, $b_{i} \geq 0(i=1,2, \ldots, m-2)$ with $\rho_{1}:=\sum_{i=1}^{m-2} b_{i} \xi_{i}^{\nu_{1}-\beta-1}<1$, and $\rho_{2}:=\sum_{i=1}^{m-2} b_{i} \xi_{i}^{\nu_{2}-\beta-1}<1$. Our analysis relies on the Banach contraction principle and Krasnoselskii's fixed point theorem.
\end{abstract}

MSC: $26 \mathrm{~A} 33 ; 34 \mathrm{~B} 18 ; 34 \mathrm{~B} 27$

Keywords: existence and uniqueness; solutions; Riemann-Liouville fractional derivative; multi-point boundary value problems; Banach contraction principle; Krasnoselskii's fixed point theorem

\section{Introduction}

Fractional calculus is a generalization of ordinary differentiation and integration to arbitrary non-integer order. The first definition of fractional derivative was introduced at the end of the nineteenth century by Liouville and Riemann, but the concept of non-integer derivative and integral, as a generalization of the traditional integer order differential and integral calculus, was mentioned already in 1695 by Leibniz and L'Hospital. With the help of fractional calculus, we can describe natural phenomena and mathematical models more accurately. The fractional differential equations play an important role in various fields of engineering, physics, economics and biological sciences, etc. (see [1-4] for example). In consequence, the subject of fractional differential equations is gaining much importance and attention. For more details on basic theory of fractional differential equations, one can see the monographs of Diethelm [1], Kilbas et al. [2], Miller and Ross [3], Podlubny [4] and Tarasov [5], and the papers [6-13] and the references therein.

(c) 2015 Zhai and Hao; licensee Springer. This article is distributed under the terms of the Creative Commons Attribution 4.0 International License (http://creativecommons.org/licenses/by/4.0/), which permits unrestricted use, distribution, and reproduction in any medium, provided you give appropriate credit to the original author(s) and the source, provide a link to the Creative Commons license, and indicate if changes were made. 
As is known to all, the initial and boundary value problems for nonlinear fractional differential equations arise from the study of models of control, porous media, electrochemistry, viscoelasticity, electromagnetics, etc. Recently, the existence and uniqueness of solutions of initial and boundary value problems for nonlinear fractional equations have been extensively studied (see [14-20]), and some are coupled systems of nonlinear fractional differential equations (see $[8,14,17,21,22])$.

In [15], Mophou studied the mild solutions to impulsive fractional differential equations

$$
\left\{\begin{array}{l}
D_{t}^{\alpha} x(t)=A x(t)+f(t, x(t)), \quad t \in I=[0, T], t \neq t_{k}, \\
x(0)=x_{0} \in X \\
\left.\Delta x\right|_{t=t_{k}}=I_{k}\left(x\left(t_{k}^{-}\right)\right), \quad k=1,2, \ldots, m
\end{array}\right.
$$

where $0<\alpha<1$, the operator $A: D(A) \subset X \rightarrow X$ is a generator of $C_{0}$-semigroup $(T(t))_{t \geq 0}$ on a Banach space $X, D_{t}^{\alpha}$ is the Caputo fractional derivative, $f: I \times X \rightarrow X$ is a given continuous function, $I_{k}: X \rightarrow X, 0=t_{0}<t_{1}<\cdots<t_{m}<t_{m+1}=T,\left.\Delta x\right|_{t=t_{k}}=x\left(t_{k}^{+}\right)-x\left(t_{k}^{-}\right)$. Some existence and uniqueness results for the equations were established by means of Krasnoselskii's fixed point theorem.

By using the same fixed point theorem, Goodrich [23] considered the existence of a positive solution to the following system of differential equations of fractional order:

$$
\left\{\begin{array}{l}
-D_{0^{+}}^{\nu_{1}} y_{1}(t)=\lambda_{1} a_{1}(t) f\left(y_{1}(t), y_{2}(t)\right) \\
-D_{0^{+}}^{\nu_{2}} y_{2}(t)=\lambda_{2} a_{2}(t) g\left(y_{1}(t), y_{2}(t)\right)
\end{array}\right.
$$

where $D_{0^{+}}^{v}$ is the standard Riemann-Liouville fractional derivative of order $v, t \in(0,1)$, $v_{1}, v_{2} \in(n-1, n]$ for $n>3$ and $n \in \mathbf{N}$, and $\lambda_{1}, \lambda_{2}>0$, with the following boundary value conditions:

$$
\begin{aligned}
& y_{1}^{(i)}(0)=0=y_{2}^{(i)}(0), \quad 0 \leq i \leq n-2, \\
& {\left[D_{0+}^{\alpha} y_{1}(t)\right]_{t=1}=0=\left[D_{0+}^{\alpha} y_{2}(t)\right]_{t=1}, \quad 1 \leq \alpha \leq n-2,}
\end{aligned}
$$

under the assumptions that $a_{1}, a_{2}, f, g$ are nonnegative and continuous.

Very recently, Sun et al. [14] considered the coupled system of multi-term nonlinear fractional differential equations

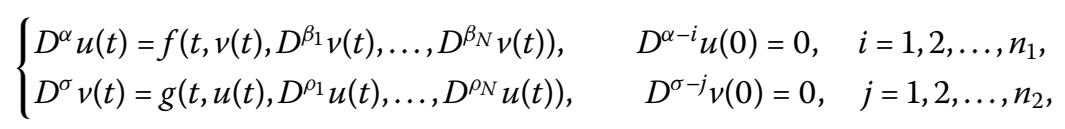

where $t \in(0,1], \alpha>\beta_{1}>\beta_{2}>\cdots>\beta_{N}>0, \sigma>\rho_{1}>\rho_{2} \cdots>\rho_{N}>0, n_{1}=[\alpha]+1, n_{2}=[\sigma]+1$, $\beta_{q}, \rho_{q}<1$ and $q \in\{1,2, \ldots, N\}$. By using the Schauder fixed point theorem and the Banach contraction principle, some results of existence and uniqueness of solutions for the coupled system are obtained.

However, to our knowledge, there are few works that deal with multi-point boundary value problems for a coupled system of nonlinear fractional differential equations. The purpose of this article is to investigate the solutions for the coupled system of nonlinear fractional differential equations (1) with the multi-point boundary conditions:

$$
y_{1}^{(i)}(0)=0=y_{2}^{(i)}(0), \quad 0 \leq i \leq n-2,
$$




$$
\begin{aligned}
& D_{0^{+}}^{\beta} y_{1}(1)=\sum_{i=1}^{m-2} b_{i} D_{0^{+}}^{\beta} y_{1}\left(\xi_{i}\right), \\
& D_{0^{+}}^{\beta} y_{2}(1)=\sum_{i=1}^{m-2} b_{i} D_{0^{+}}^{\beta} y_{2}\left(\xi_{i}\right),
\end{aligned}
$$

where $n-2<\beta<n-1,0<\xi_{1}<\xi_{2}<\cdots<\xi_{m-2}<1, b_{i} \geq 0(i=1,2, \ldots, m-2)$ with $\rho_{1}:=$ $\sum_{i=1}^{m-2} b_{i} \xi_{i}^{\nu_{1}-\beta-1}<1$, and $\rho_{2}:=\sum_{i=1}^{m-2} b_{i} \xi_{i}^{\nu_{2}-\beta-1}<1$.

Motivated by the above-mentioned works and recent works on coupled systems of fractional differential equations, we consider the existence and uniqueness of solutions of coupled system (1)-(4) by means of the Banach contraction principle and Krasnoselskii's fixed point theorem. In our paper, we do not suppose that $a_{1}, a_{2}, f, g$ are nonnegative.

With this context in mind, the outline of this paper is as follows. In Section 2 we recall certain results from the theory of continuous fractional calculus. In Section 3 we provide some conditions under which problem (1)-(4) will have a unique solution or at least one solution.

\section{Preliminaries}

For the convenience of the reader, we present here some definitions, lemmas and basic results that will be used in the proofs of our theorems.

Definition 2.1 (see [4]) Let $v>0$ with $v \in \mathbf{R}$. Suppose that $y:[a,+\infty) \rightarrow \mathbf{R}$. Then the $v$ th Riemann-Liouville fractional integral is defined to be

$$
D_{a^{+}}^{-v} y(t):=\frac{1}{\Gamma(v)} \int_{a}^{t} y(s)(t-s)^{\nu-1} d s
$$

whenever the right-hand side is defined. Similarly, with $v>0$ and $v \in \mathbf{R}$, we define the $v$ th Riemann-Liouville fractional derivative to be

$$
D_{a^{+}}^{v} y(t):=\frac{1}{\Gamma(n-v)} \frac{d^{n}}{d t^{n}} \int_{a}^{t} \frac{y(s)}{(t-s)^{v+1-n}} d s,
$$

where $n \in \mathbf{N}$ is the unique positive integer satisfying $n-1 \leq v<n$ and $t>a$.

Lemma 2.2 (see [23]) Let $\alpha \in \mathbf{R}$. Then $D^{n} D_{a^{+}}^{\alpha} y(t)=D_{a^{+}}^{n+\alpha} y(t)$, for each $n \in N_{0}$, where $y(t)$ is assumed to be sufficiently regular so that both sides of the equality are well defined. Moreover, if $\beta \in(-\infty, 0]$ and $\gamma \in[0,+\infty)$, then $D_{a^{+}}^{\gamma} D_{a^{+}}^{\beta} y(t)=D_{a^{+}}^{\gamma+\beta} y(t)$.

Lemma 2.3 (see [23]) The general solution to $D_{a^{+}}^{v} y(t)=0$, where $n-1<v \leq n$ and $v>0$, is the function $y(t)=c_{1} t^{\nu-1}+c_{2} t^{\nu-2}+\cdots+c_{n} t^{\nu-n}$, where $c_{i} \in \mathbf{R}$ for each $i$.

Lemma 2.4 Let $h \in C^{n}([0,1])$ be given. Then the unique solution to problem $-D_{0^{+}}^{v} y(t)=$ $h(t)$ together with the boundary conditions $y^{(i)}(0)=0$ and $D_{0^{+}}^{\beta} y(1)=\sum_{i=1}^{m-2} b_{i} D_{0^{+}}^{\beta} y\left(\xi_{i}\right)$, where $n-2<\beta<n-1$ and $0 \leq i \leq n-2$, is

$$
y(t)=\int_{0}^{1} G(t, s) h(s) d s
$$


where

$$
G(t, s)=\left\{\begin{array}{l}
-\frac{(t-s)^{\nu-1}}{\Gamma(\nu)}+\frac{t^{\nu-1}}{\Gamma(v)(1-\rho)}\left[(1-s)^{\nu-\beta-1}-\sum_{i=1}^{m-2} b_{i}\left(\xi_{i}-s\right)^{\nu-\beta-1}\right] \\
s \leq t, \xi_{i-1}<s \leq \xi_{i}, i=1,2, \ldots m-1 \\
\frac{t^{\nu-1}}{\Gamma(\nu)(1-\rho)}\left[(1-s)^{\nu-\beta-1}-\sum_{i=1}^{m-2} b_{i}\left(\xi_{i}-s\right)^{\nu-\beta-1}\right] \\
t \leq s, \xi_{i-1}<s \leq \xi_{i}, i=1,2, \ldots m-1
\end{array}\right.
$$

is the Green function for this problem, where $\rho=\sum_{i=1}^{m-2} b_{i} \xi_{i}^{\nu-\beta-1}<1$ and $\xi_{0}=0, \xi_{m-1}=1$.

Proof We know that the general solution to our problem is

$$
y(t)=c_{1} t^{\nu-1}+c_{2} t^{\nu-2}+\cdots+c_{n} t^{\nu-n}-D_{0^{+}}^{-\nu} h(t)
$$

we immediately observe that the boundary value condition $y^{(i)}(0)=0,0 \leq i \leq n-2$, implies that $c_{2}=c_{3}=\cdots=c_{n}=0$. On the other hand, $D_{0^{+}}^{\beta} y(1)=\sum_{i=1}^{m-2} b_{i} D_{0^{+}}^{\beta} y\left(\xi_{i}\right)$ implies that

$$
\begin{aligned}
- & \frac{1}{\Gamma(v-\beta)} \int_{0}^{1}(1-s)^{v-\beta-1} h(s) d s+c_{1} \frac{\Gamma(v)}{\Gamma(v-\beta)} \\
& =\sum_{i=1}^{m-2} b_{i}\left(-\frac{1}{\Gamma(v-\beta)} \int_{0}^{\xi_{i}}\left(\xi_{i}-s\right)^{v-\beta-1} h(s) d s+c_{1} \frac{\Gamma(v)}{\Gamma(v-\beta)} \xi_{i}^{v-\beta-1}\right) .
\end{aligned}
$$

That is to say,

$$
c_{1}=\frac{1}{\Gamma(v)(1-\rho)}\left[\int_{0}^{1}(1-s)^{\nu-\beta-1} h(s) d s-\sum_{i=1}^{m-2} b_{i} \int_{0}^{\xi_{i}}\left(\xi_{i}-s\right)^{\nu-\beta-1} h(s) d s\right] .
$$

Therefore, the unique solution is

$$
\begin{aligned}
y(t)= & -\int_{0}^{t} \frac{(t-s)^{\nu-1}}{\Gamma(v)} h(s) d s \\
& +\frac{t^{\nu-1}}{\Gamma(\nu)(1-\rho)}\left[\int_{0}^{1}(1-s)^{\nu-\beta-1} h(s) d s-\sum_{i=1}^{m-2} b_{i} \int_{0}^{\xi_{i}}\left(\xi_{i}-s\right)^{\nu-\beta-1} h(s) d s\right] \\
= & \int_{0}^{1} G(t, s) h(s) d s .
\end{aligned}
$$

The proof is complete.

\section{Main results}

This section deals with the existence and uniqueness of solutions to problem (1)-(4).

Let $E$ represent the Banach space of $C[0,1]$ when equipped with the usual supremum norm $\|\cdot\|$. Then put $X:=E \times E$, where $X$ is equipped with the norm

$$
\left\|\left(y_{1}, y_{2}\right)\right\|:=\left\|y_{1}\right\|+\left\|y_{2}\right\|
$$


for $\left(y_{1}, y_{2}\right) \in X$. Observe that $X$ is also a Banach space (see [24]). In addition, define two operators $T_{1}, T_{2}: X \rightarrow E$ by

$$
\left(T_{1}\left(y_{1}, y_{2}\right)\right)(t):=\lambda_{1} \int_{0}^{1} G_{1}(t, s) a_{1}(s) f\left(s, y_{1}(s), y_{2}(s)\right) d s
$$

and

$$
\left(T_{2}\left(y_{1}, y_{2}\right)\right)(t):=\lambda_{2} \int_{0}^{1} G_{2}(t, s) a_{2}(s) g\left(s, y_{1}(s), y_{2}(s)\right) d s,
$$

where $G_{1}(t, s)$ is the Green function of Lemma 2.4 with $v$ replaced by $v_{1}$ and $\rho$ replaced by $\rho_{1}$, and likewise, $G_{2}(t, s)$ is the Green function of Lemma 2.4 with $v$ replaced by $v_{2}$ and $\rho$ replaced by $\rho_{2}$. Now, we define an operator $S: X \rightarrow X$ by

$$
\begin{aligned}
\left(S\left(y_{1}, y_{2}\right)\right)(t):= & \left(\left(T_{1}\left(y_{1}, y_{2}\right)\right)(t),\left(T_{2}\left(y_{1}, y_{2}\right)\right)(t)\right) \\
= & \left(\lambda_{1} \int_{0}^{1} G_{1}(t, s) a_{1}(s) f\left(s, y_{1}(s), y_{2}(s)\right) d s,\right. \\
& \left.\lambda_{2} \int_{0}^{1} G_{2}(t, s) a_{2}(s) g\left(s, y_{1}(s), y_{2}(s)\right) d s\right) .
\end{aligned}
$$

We claim that whenever $\left(y_{1}, y_{2}\right) \in X$ is a fixed point of the operator defined in (7), it follows that $y_{1}(t)$ and $y_{2}(t)$ solve problem (1)-(4). We shall look for fixed points of the operator $S$, seeing as these fixed points coincide with solutions of problem (1)-(4).

To establish the main results, we need the following assumptions:

$\left(\mathrm{H}_{1}\right) f, g:[0,1] \times \mathbf{R} \times \mathbf{R} \rightarrow \mathbf{R}$ and $a_{1}, a_{2}:[0,1] \rightarrow \mathbf{R} ;$

$\left(\mathrm{H}_{2}\right) f, g, a_{1}, a_{2}$ are continuous;

$\left(\mathrm{H}_{3}\right)$ there exist positive functions $L_{1}(t)$ and $L_{2}(t)$ such that

$$
\begin{aligned}
& \left|a_{1}(t)\right| \cdot\left|f\left(t, y_{1}(t), y_{2}(t)\right)-f\left(t, u_{1}(t), u_{2}(t)\right)\right| \leq L_{1}(t)\left\|\left(y_{1}-u_{1}, y_{2}-u_{2}\right)\right\|, \\
& \left|a_{2}(t)\right| \cdot\left|g\left(t, y_{1}(t), y_{2}(t)\right)-g\left(t, u_{1}(t), u_{2}(t)\right)\right| \leq L_{2}(t)\left\|\left(y_{1}-u_{1}, y_{2}-u_{2}\right)\right\|
\end{aligned}
$$

for all $t \in[0,1]$ and $\left(y_{1}, y_{2}\right),\left(u_{1}, u_{2}\right) \in X$.

Further, we set

$$
\begin{aligned}
& D_{L}^{-\nu}=\max \left\{\sup _{t \in[0,1]}\left|D_{0^{+}}^{-\nu_{1}} L_{1}(t)\right|, \sup _{t \in[0,1]}\left|D_{0^{+}}^{-\nu_{2}} L_{2}(t)\right|\right\}, \\
& D^{-(\nu-\beta)} L(1)=\max \left\{\left|D_{0^{+}}^{-\left(\nu_{1}-\beta\right)} L_{1}(1)\right|,\left|D_{0^{+}}^{-\left(\nu_{2}-\beta\right)} L_{2}(1)\right|\right\}, \\
& D^{-(\nu-\beta)} L\left(\xi_{i}\right)=\max \left\{\left|D_{0^{+}}^{-\left(\nu_{1}-\beta\right)} L_{1}\left(\xi_{i}\right)\right|,\left|D_{0^{+}}^{-\left(\nu_{2}-\beta\right)} L_{2}\left(\xi_{i}\right)\right|\right\}, \quad i=1,2, \ldots, m-2 .
\end{aligned}
$$

$\left(\mathrm{H}_{4}\right)$ The parameters $\lambda_{1}, \lambda_{2}$ satisfy $\lambda_{1}, \lambda_{2}<\Lambda$, where

$$
\begin{aligned}
\Lambda= & \min \left\{\frac{1}{4}\left[D_{L}^{-v}+\frac{\Gamma\left(v_{1}-\beta\right)}{\Gamma\left(v_{1}\right)\left(1-\rho_{1}\right)}\left(\sum_{i=1}^{m-2} b_{i} D^{-(v-\beta)} L\left(\xi_{i}\right)+D^{-(\nu-\beta)} L(1)\right)\right]^{-1},\right. \\
& \left.\frac{1}{4}\left[D_{L}^{-\nu}+\frac{\Gamma\left(v_{2}-\beta\right)}{\Gamma\left(v_{2}\right)\left(1-\rho_{2}\right)}\left(\sum_{i=1}^{m-2} b_{i} D^{-(\nu-\beta)} L\left(\xi_{i}\right)+D^{-(\nu-\beta)} L(1)\right)\right]^{-1}\right\} .
\end{aligned}
$$


$\left(\mathrm{H}_{5}\right)$ The parameters $\lambda_{1}, \lambda_{2}$ satisfy $\lambda_{1}, \lambda_{2}<\Lambda$, where

$$
\begin{aligned}
\Lambda= & \min \left\{\frac{1}{4}\left[\frac{\Gamma\left(v_{1}-\beta\right)}{\Gamma\left(v_{1}\right)\left(1-\rho_{1}\right)}\left(\sum_{i=1}^{m-2} b_{i} D^{-(\nu-\beta)} L\left(\xi_{i}\right)+D^{-(\nu-\beta)} L(1)\right)\right]^{-1},\right. \\
& \left.\frac{1}{4}\left[\frac{\Gamma\left(v_{2}-\beta\right)}{\Gamma\left(v_{2}\right)\left(1-\rho_{2}\right)}\left(\sum_{i=1}^{m-2} b_{i} D^{-(v-\beta)} L\left(\xi_{i}\right)+D^{-(v-\beta)} L(1)\right)\right]^{-1}\right\} .
\end{aligned}
$$

$\left(\mathrm{H}_{6}\right)$ There exists $\mu \in L^{1}\left([0,1], \mathbf{R}^{+}\right)$such that

$$
\begin{aligned}
& \left|a_{1}(t) f\left(t, y_{1}(t), y_{2}(t)\right)\right| \leq \mu(t), \quad\left|a_{2}(t) g\left(t, y_{1}(t), y_{2}(t)\right)\right| \leq \mu(t), \\
& \forall\left(t, y_{1}, y_{2}\right) \in[0,1] \times X .
\end{aligned}
$$

We are ready to state the existence and uniqueness result.

Theorem 3.1 Suppose that conditions $\left(\mathrm{H}_{1}\right)-\left(\mathrm{H}_{4}\right)$ are satisfied. Then the boundary value problem (1)-(4) has a unique solution.

Proof Let us set

$$
M=\max \left\{\sup _{t \in[0,1]}\left|a_{1}(t) f(t, 0,0)\right|, \sup _{t \in[0,1]}\left|a_{2}(t) g(t, 0,0)\right|\right\}
$$

and choose

$$
\begin{gathered}
r \geq \max \left\{4 \lambda_{1}\left[\frac{M}{\Gamma\left(v_{1}+1\right)}+\frac{M}{\Gamma\left(v_{1}\right)\left(1-\rho_{1}\right)\left(v_{1}-\beta\right)}\left(\sum_{i=1}^{m-2} b_{i} \xi_{i}^{\nu_{1}-\beta}+1\right)\right],\right. \\
\left.\quad 4 \lambda_{2}\left[\frac{M}{\Gamma\left(v_{2}+1\right)}+\frac{M}{\Gamma\left(v_{2}\right)\left(1-\rho_{2}\right)\left(v_{2}-\beta\right)}\left(\sum_{i=1}^{m-2} b_{i} \xi_{i}^{\nu_{2}-\beta}+1\right)\right]\right\} .
\end{gathered}
$$

Now, we show that $S\left(\Omega_{r}\right) \subset \Omega_{r}$, where $\Omega_{r}=\left\{\left(y_{1}, y_{2}\right) \in X:\left\|\left(y_{1}, y_{2}\right)\right\| \leq r\right\}$, and $S$ is a contraction. In fact, for all $\left(y_{1}, y_{2}\right) \in \Omega_{r}$, we obtain

$$
\begin{aligned}
& \left\|T_{1}\left(y_{1}, y_{2}\right)\right\| \\
& =\max _{t \in[0,1]} \mid-\int_{0}^{t} \frac{(t-s)^{\nu_{1}-1}}{\Gamma\left(v_{1}\right)} \lambda_{1} a_{1}(s) f\left(s, y_{1}(s), y_{2}(s)\right) d s \\
& \quad+\frac{t^{\nu_{1}-1}}{\Gamma\left(\nu_{1}\right)\left(1-\rho_{1}\right)}\left[\int_{0}^{1}(1-s)^{\nu_{1}-\beta-1} \lambda_{1} a_{1}(s) f\left(s, y_{1}(s), y_{2}(s)\right) d s\right. \\
& \left.\quad-\sum_{i=1}^{m-2} b_{i} \int_{0}^{\xi_{i}}\left(\xi_{i}-s\right)^{\nu_{1}-\beta-1} \lambda_{1} a_{1}(s) f\left(s, y_{1}(s), y_{2}(s)\right) d s\right] \mid \\
& \leq \max _{t \in[0,1]}\left\{\int_{0}^{t} \frac{(t-s)^{\nu_{1}-1}}{\Gamma\left(v_{1}\right)} \lambda_{1}\left|a_{1}(s) f\left(s, y_{1}(s), y_{2}(s)\right)\right| d s\right. \\
& \quad+\frac{t^{\nu_{1}-1}}{\Gamma\left(v_{1}\right)\left(1-\rho_{1}\right)}\left[\int_{0}^{1}(1-s)^{\nu_{1}-\beta-1} \lambda_{1}\left|a_{1}(s) f\left(s, y_{1}(s), y_{2}(s)\right)\right| d s\right.
\end{aligned}
$$




$$
\begin{aligned}
& \left.\left.+\sum_{i=1}^{m-2} b_{i} \int_{0}^{\xi_{i}}\left(\xi_{i}-s\right)^{\nu_{1}-\beta-1} \lambda_{1}\left|a_{1}(s) f\left(s, y_{1}(s), y_{2}(s)\right)\right| d s\right]\right\} \\
& \leq \max _{t \in[0,1]}\left\{\int_{0}^{t} \frac{(t-s)^{\nu_{1}-1}}{\Gamma\left(v_{1}\right)} \lambda_{1}\left|a_{1}(s)\right|\left(\left|f\left(s, y_{1}(s), y_{2}(s)\right)-f(s, 0,0)\right|+|f(s, 0,0)|\right) d s\right. \\
& +\frac{t^{\nu_{1}-1}}{\Gamma\left(v_{1}\right)\left(1-\rho_{1}\right)}\left[\int _ { 0 } ^ { 1 } ( 1 - s ) ^ { \nu _ { 1 } - \beta - 1 } \lambda _ { 1 } | a _ { 1 } ( s ) | \left(\left|f\left(s, y_{1}(s), y_{2}(s)\right)-f(s, 0,0)\right|\right.\right. \\
& +|f(s, 0,0)|) d s \\
& \left.\left.+\sum_{i=1}^{m-2} b_{i} \int_{0}^{\xi_{i}}\left(\xi_{i}-s\right)^{\nu_{1}-\beta-1} \lambda_{1}\left|a_{1}(s)\right|\left(\left|f\left(s, y_{1}(s), y_{2}(s)\right)-f(s, 0,0)\right|+|f(s, 0,0)|\right) d s\right]\right\} \\
& \leq \max _{t \in[0,1]}\left\{\int_{0}^{t} \frac{(t-s)^{v_{1}-1}}{\Gamma\left(v_{1}\right)} \lambda_{1}\left(L_{1}(s)\left\|\left(y_{1}, y_{2}\right)\right\|+M\right) d s\right. \\
& +\frac{t^{\nu_{1}-1}}{\Gamma\left(v_{1}\right)\left(1-\rho_{1}\right)}\left[\int_{0}^{1}(1-s)^{\nu_{1}-\beta-1} \lambda_{1}\left(L_{1}(s)\left\|\left(y_{1}, y_{2}\right)\right\|+M\right) d s\right. \\
& \left.\left.+\sum_{i=1}^{m-2} b_{i} \int_{0}^{\xi_{i}}\left(\xi_{i}-s\right)^{\nu_{1}-\beta-1} \lambda_{1}\left(L_{1}(s)\left\|\left(y_{1}, y_{2}\right)\right\|+M\right) d s\right]\right\} \\
& \leq \max _{t \in[0,1]}\left\{\lambda_{1}\left(D_{0^{+}}^{-\nu_{1}} L_{1}(t) r+\frac{M t^{\nu_{1}}}{\Gamma\left(v_{1}+1\right)}\right)+\lambda_{1} \frac{\Gamma\left(\nu_{1}-\beta\right) t^{\nu_{1}-1}}{\Gamma\left(\nu_{1}\right)\left(1-\rho_{1}\right)}\right. \\
& \left.\times\left[\sum_{i=1}^{m-2} b_{i}\left(D_{0^{+}}^{-\left(v_{1}-\beta\right)} L_{1}\left(\xi_{i}\right) r+\frac{M \xi_{i}^{\nu_{1}-\beta}}{\Gamma\left(v_{1}-\beta+1\right)}\right)+D_{0^{+}}^{-\left(v_{1}-\beta\right)} L_{1}(1) r+\frac{M}{\Gamma\left(v_{1}-\beta+1\right)}\right]\right\} \\
& \leq \lambda_{1}\left(D_{L}^{-v} r+\frac{M}{\Gamma\left(v_{1}+1\right)}\right)+\lambda_{1} \frac{\Gamma\left(v_{1}-\beta\right)}{\Gamma\left(v_{1}\right)\left(1-\rho_{1}\right)} \\
& \times\left[\sum_{i=1}^{m-2} b_{i}\left(D^{-(v-\beta)} L\left(\xi_{i}\right) r+\frac{M \xi_{i}^{\nu_{1}-\beta}}{\Gamma\left(v_{1}-\beta+1\right)}\right)+D^{-(v-\beta)} L(1) r+\frac{M}{\Gamma\left(v_{1}-\beta+1\right)}\right] \\
& \leq \lambda_{1}\left[D_{L}^{-v}+\frac{\Gamma\left(v_{1}-\beta\right)}{\Gamma\left(v_{1}\right)\left(1-\rho_{1}\right)}\left(\sum_{i=1}^{m-2} b_{i} D^{-(v-\beta)} L\left(\xi_{i}\right)+D^{-(v-\beta)} L(1)\right)\right] r \\
& +\lambda_{1} M\left[\frac{1}{\Gamma\left(v_{1}+1\right)}+\frac{1}{\Gamma\left(v_{1}\right)\left(1-\rho_{1}\right)\left(\nu_{1}-\beta\right)}\left(\sum_{i=1}^{m-2} b_{i} \xi_{i}^{\nu_{1}-\beta}+1\right)\right] \\
& \leq \frac{1}{4} r+\frac{1}{4} r=\frac{r}{2} \text {, }
\end{aligned}
$$

that is to say, $\left\|T_{1}\left(y_{1}, y_{2}\right)\right\| \leq \frac{r}{2}$.

Then, for $\left(y_{1}, y_{2}\right),\left(u_{1}, u_{2}\right) \in X$ and for each $t \in[0,1]$, we obtain

$$
\begin{aligned}
& \left\|T_{1}\left(y_{1}, y_{2}\right)-T_{1}\left(u_{1}, u_{2}\right)\right\| \\
& \leq \max _{t \in[0,1]}\left\{\int_{0}^{t} \frac{(t-s)^{\nu_{1}-1}}{\Gamma\left(v_{1}\right)} \lambda_{1}\left|a_{1}(s)\right| \cdot\left|f\left(s, y_{1}(s), y_{2}(s)\right)-f\left(s, u_{1}(s), u_{2}(s)\right)\right| d s\right. \\
& \quad+\frac{t^{\nu_{1}-1}}{\Gamma\left(v_{1}\right)\left(1-\rho_{1}\right)}\left[\int_{0}^{1}(1-s)^{\nu_{1}-\beta-1} \lambda_{1}\left|a_{1}(s)\right|\right.
\end{aligned}
$$




$$
\begin{aligned}
& \times\left|f\left(s, y_{1}(s), y_{2}(s)\right)-f\left(s, u_{1}(s), u_{2}(s)\right)\right| d s \\
& \left.\left.+\sum_{i=1}^{m-2} b_{i} \int_{0}^{\xi_{i}}\left(\xi_{i}-s\right)^{\nu_{1}-\beta-1} \lambda_{1}\left|a_{1}(s)\right| \cdot\left|f\left(s, y_{1}(s), y_{2}(s)\right)-f\left(s, u_{1}(s), u_{2}(s)\right)\right| d s\right]\right\} \\
\leq & \lambda_{1} D_{L}^{-v}\left\|\left(y_{1}-u_{1}, y_{2}-u_{2}\right)\right\|+\lambda_{1} \frac{\Gamma\left(v_{1}-\beta\right)}{\Gamma\left(v_{1}\right)\left(1-\rho_{1}\right)} \\
& \times\left[\sum_{i=1}^{m-2} b_{i} D^{-\left(v_{1}-\beta\right)} L\left(\xi_{i}\right)+D^{-\left(v_{1}-\beta\right)} L(1)\right]\left\|\left(y_{1}-u_{1}, y_{2}-u_{2}\right)\right\| \\
\leq & \frac{1}{4}\left\|\left(y_{1}-u_{1}, y_{2}-u_{2}\right)\right\| .
\end{aligned}
$$

That is to say, $\left\|T_{1}\left(y_{1}, y_{2}\right)-T_{1}\left(u_{1}, u_{2}\right)\right\| \leq \frac{1}{4}\left\|\left(y_{1}-u_{1}, y_{2}-u_{2}\right)\right\|=\frac{1}{4}\left\|\left(y_{1}, y_{2}\right)-\left(u_{1}, u_{2}\right)\right\|$.

Hence, we find that $T_{1}: \Omega_{r} \rightarrow B_{\frac{r}{2}}$ and $T_{1}$ is a contraction, where $B_{\frac{r}{2}}=\left\{y \in B:\|y\| \leq \frac{r}{2}\right\}$. Similarly, we have $T_{2}: \Omega_{r} \rightarrow B_{\frac{r}{2}}$ and $T_{2}$ is a contraction. Consequently, for any $\left(y_{1}, y_{2}\right) \in$ $\Omega_{r}$,

$$
\left\|S\left(y_{1}, y_{2}\right)\right\|=\left\|\left(T_{1}\left(y_{1}, y_{2}\right), T_{2}\left(y_{1}, y_{2}\right)\right)\right\| \leq \frac{r}{2}+\frac{r}{2} \leq r
$$

i.e., $S\left(\Omega_{r}\right) \subset \Omega_{r}$. And, for $\left(y_{1}, y_{2}\right),\left(u_{1}, u_{2}\right) \in \Omega_{r}$ and for each $t \in[0,1]$,

$$
\left\|S\left(y_{1}, y_{2}\right)-S\left(u_{1}, u_{2}\right)\right\| \leq \frac{1}{2}\left\|\left(y_{1}-u_{1}, y_{2}-u_{2}\right)\right\|=\frac{1}{2}\left\|\left(y_{1}, y_{2}\right)-\left(u_{1}, u_{2}\right)\right\| .
$$

So, $S: \Omega_{r} \rightarrow \Omega_{r}$ and $S$ is a contraction. Thus, the conclusion of the theorem follows from the contraction mapping principle.

Our next result is based on the following well-known fixed point theorem due to Krasnoselskii.

Lemma 3.2 (Krasnoselskii [25]) Let $K$ be a closed convex and nonempty subset of a Banach space E. Let $T, S$ be the operators such that:

(i) $T x+S y \in K$ whenever $x, y \in K$;

(ii) $T$ is compact and continuous;

(iii) $S$ is a contraction mapping.

Then there exists $z \in K$ such that $z=T z+S z$.

Now we are ready to state and prove the following existence result.

Theorem 3.3 Suppose that conditions $\left(\mathrm{H}_{1}\right)-\left(\mathrm{H}_{3}\right),\left(\mathrm{H}_{5}\right),\left(\mathrm{H}_{6}\right)$ are satisfied. Then there exists at least one solution of the boundary value problem (1)-(4).

Proof Let us fix

$$
\begin{gathered}
r \geq \max \left\{\frac{1}{2} \lambda_{1}\|\mu\|_{L^{1}}\left[\frac{1}{\Gamma\left(v_{1}+1\right)}+\frac{1}{\Gamma\left(v_{1}\right)\left(1-\rho_{1}\right)\left(v_{1}-\beta\right)}\left(\sum_{i=1}^{m-2} b_{i} \xi_{i}^{v_{1}-\beta}+1\right)\right],\right. \\
\left.\frac{1}{2} \lambda_{2}\|\mu\|_{L^{1}}\left[\frac{1}{\Gamma\left(v_{2}+1\right)}+\frac{1}{\Gamma\left(v_{2}\right)\left(1-\rho_{2}\right)\left(v_{2}-\beta\right)}\left(\sum_{i=1}^{m-2} b_{i} \xi_{i}^{v_{2}-\beta}+1\right)\right]\right\},
\end{gathered}
$$


and consider $\Omega_{r}=\left\{\left(y_{1}, y_{2}\right) \in X:\left\|\left(y_{1}, y_{2}\right)\right\| \leq r\right\}$. We define the operators $Q_{1}$ and $Q_{2}$ on $\Omega_{r}$ as

$$
T_{1}\left(y_{1}, y_{2}\right):=Q_{1}\left(y_{1}, y_{2}\right)+Q_{2}\left(y_{1}, y_{2}\right)
$$

where

$$
\begin{aligned}
\left(Q_{1}\left(y_{1}, y_{2}\right)\right)(t)= & -\int_{0}^{t} \frac{(t-s)^{\nu_{1}-1}}{\Gamma\left(v_{1}\right)} \lambda_{1} a_{1}(s) f\left(s, y_{1}(s), y_{2}(s)\right) d s \\
\left(Q_{2}\left(y_{1}, y_{2}\right)\right)(t)= & \frac{t^{\nu_{1}-1}}{\Gamma\left(\nu_{1}\right)\left(1-\rho_{1}\right)}\left[\int_{0}^{1}(1-s)^{\nu_{1}-\beta-1} \lambda_{1} a_{1}(s) f\left(s, y_{1}(s), y_{2}(s)\right) d s\right. \\
& \left.-\sum_{i=1}^{m-2} b_{i} \int_{0}^{\xi_{i}}\left(\xi_{i}-s\right)^{\nu_{1}-\beta-1} \lambda_{1} a_{1}(s) f\left(s, y_{1}(s), y_{2}(s)\right) d s\right]
\end{aligned}
$$

For all $\left(y_{1}, y_{2}\right),\left(u_{1}, u_{2}\right) \in \Omega_{r}$, we find that

$$
\begin{aligned}
& \left\|Q_{1}\left(y_{1}, y_{2}\right)+Q_{2}\left(u_{1}, u_{2}\right)\right\| \\
& \quad \leq\|\mu\|_{L^{1}} \lambda_{1}\left[\frac{1}{\Gamma\left(v_{1}+1\right)}+\frac{1}{\Gamma\left(v_{1}\right)\left(1-\rho_{1}\right)\left(v_{1}-\beta\right)}\left(\sum_{i=1}^{m-2} b_{i} \xi_{i}^{\nu_{1}-\beta}+1\right)\right] \\
& \quad \leq \frac{r}{2} .
\end{aligned}
$$

Thus, $Q_{1}\left(y_{1}, y_{2}\right)+Q_{2}\left(u_{1}, u_{2}\right) \in \Omega_{\frac{r}{2}}$ for all $\left(y_{1}, y_{2}\right),\left(u_{1}, u_{2}\right) \in \Omega_{r}$. From assumption $\left(\mathrm{H}_{3}\right)$, we have

$$
\begin{aligned}
\left\|Q_{2}\left(y_{1}, y_{2}\right)-Q_{2}\left(u_{1}, u_{2}\right)\right\| \leq & \lambda_{1} \frac{\Gamma\left(v_{1}-\beta\right)}{\Gamma\left(v_{1}\right)\left(1-\rho_{1}\right)}\left[\sum_{i=1}^{m-2} b_{i} D^{-\left(\nu_{1}-\beta\right)} L\left(\xi_{i}\right)+D^{-\left(v_{1}-\beta\right)} L(1)\right] \\
& \times\left\|\left(y_{1}-u_{1}, y_{2}-u_{2}\right)\right\| \\
\leq & \frac{1}{4}\left\|\left(y_{1}-u_{1}, y_{2}-u_{2}\right)\right\| \\
& =\frac{1}{4}\left\|\left(y_{1}, y_{2}\right)-\left(u_{1}, u_{2}\right)\right\|,
\end{aligned}
$$

where $\left(y_{1}, y_{2}\right),\left(u_{1}, u_{2}\right) \in X, t \in[0,1]$. So, $Q_{2}$ is a contraction mapping. We next consider the operator $Q_{1}$. Evidently, the continuity of $f$ implies that the operator $Q_{1}$ is continuous. Also, $Q_{1}$ is uniformly bounded on $\Omega_{r}$ as

$$
\left\|Q_{1}\left(y_{1}, y_{2}\right)\right\| \leq \lambda_{1} \frac{\|\mu\|_{L^{1}}}{\Gamma\left(v_{1}+1\right)} \leq 2 r
$$

Now, we show that $Q_{1}\left(y_{1}, y_{2}\right)(t)$ is equicontinuous. In fact, since $a_{1}, f$ are bounded on the compact set $[0,1]$ and $[0,1] \times \Omega_{r}$, respectively, we can define

$$
M_{1}=\max \left\{\sup _{\left(t, y_{1}, y_{2}\right) \in[0,1] \times \Omega_{r}}\left|a_{1}(t) f\left(t, y_{1}, y_{2}\right)\right|, \sup _{\left(t, y_{1}, y_{2}\right) \in[0,1] \times \Omega_{r}}\left|a_{2}(t) g\left(t, y_{1}, y_{2}\right)\right|\right\},
$$


and we have, for any $t_{1}, t_{2} \in[0,1]$,

$$
\begin{aligned}
\left|Q_{1}\left(y_{1}, y_{2}\right)\left(t_{2}\right)-Q_{1}\left(y_{1}, y_{2}\right)\left(t_{1}\right)\right| \\
=\mid-\int_{0}^{t_{2}} \frac{\left(t_{2}-s\right)^{\nu_{1}-1}}{\Gamma\left(v_{1}\right)} \lambda_{1} a_{1}(s) f\left(s, y_{1}(s), y_{2}(s)\right) d s \\
\quad+\int_{0}^{t_{1}} \frac{\left(t_{1}-s\right)^{\nu_{1}-1}}{\Gamma\left(v_{1}\right)} \lambda_{1} a_{1}(s) f\left(s, y_{1}(s), y_{2}(s)\right) d s \mid \\
=\mid \int_{0}^{t_{1}} \frac{\left(t_{2}-s\right)^{\nu_{1}-1}-\left(t_{1}-s\right)^{\nu_{1}-1}}{\Gamma\left(v_{1}\right)} \lambda_{1} a_{1}(s) f\left(s, y_{1}(s), y_{2}(s)\right) d s \\
\quad+\int_{t_{1}}^{t_{2}} \frac{\left(t_{2}-s\right)^{\nu_{1}-1}}{\Gamma\left(v_{1}\right)} \lambda_{1} a_{1}(s) f\left(s, y_{1}(s), y_{2}(s)\right) d s \mid \\
\leq \lambda_{1} \frac{M_{1}}{\Gamma\left(v_{1}\right)}\left|\int_{0}^{t_{1}}\left(\left(t_{2}-s\right)^{\nu_{1}-1}-\left(t_{1}-s\right)^{\nu_{1}-1}\right) d s+\int_{t_{1}}^{t_{2}}\left(t_{2}-s\right)^{\nu_{1}-1} d s\right| \\
=\lambda_{1} \frac{M_{1}}{\Gamma\left(v_{1}+1\right)}\left|-\left(\left(t_{2}-t_{1}\right)^{\nu_{1}}-t_{2}^{\nu_{1}}\right)-t_{1}^{\nu_{1}}+\left(t_{2}-t_{1}\right)^{\nu_{1}}\right| \\
=\lambda_{1} \frac{M_{1}}{\Gamma\left(v_{1}+1\right)}\left|t_{2}^{\nu_{1}}-t_{1}^{\nu_{1}}\right|,
\end{aligned}
$$

which is independent of $\left(y_{1}, y_{2}\right)$. Therefore, $Q_{1}$ is equicontinuous on $\Omega_{r}$. Hence, by the Arzela-Ascoli theorem, $Q_{1}$ is compact on $\Omega_{r}$. Similarly, we set

$$
T_{2}\left(y_{1}, y_{2}\right):=P_{1}\left(y_{1}, y_{2}\right)+P_{2}\left(y_{1}, y_{2}\right),
$$

where

$$
\begin{aligned}
\left(P_{1}\left(y_{1}, y_{2}\right)\right)(t)= & -\int_{0}^{t} \frac{(t-s)^{\nu_{2}-1}}{\Gamma\left(v_{2}\right)} \lambda_{2} a_{2}(s) g\left(s, y_{1}(s), y_{2}(s)\right) d s \\
\left(P_{2}\left(y_{1}, y_{2}\right)\right)(t)= & \frac{t^{\nu_{2}-1}}{\Gamma\left(v_{2}\right)\left(1-\rho_{2}\right)}\left[\int_{0}^{1}(1-s)^{\nu_{2}-\beta-1} \lambda_{2} a_{2}(s) g\left(s, y_{1}(s), y_{2}(s)\right) d s\right. \\
& \left.-\sum_{i=1}^{m-2} b_{i} \int_{0}^{\xi_{i}}\left(\xi_{i}-s\right)^{\nu_{2}-\beta-1} \lambda_{2} a_{2}(s) g\left(s, y_{1}(s), y_{2}(s)\right) d s\right]
\end{aligned}
$$

And we can obtain a similar conclusion to the operator $T_{1}$. Now, we let

$$
A\left(y_{1}, y_{2}\right):=\left(Q_{1}\left(y_{1}, y_{2}\right), P_{1}\left(y_{1}, y_{2}\right)\right), \quad B\left(y_{1}, y_{2}\right):=\left(Q_{2}\left(y_{1}, y_{2}\right), P_{2}\left(y_{1}, y_{2}\right)\right) .
$$

Therefore, we have

$$
\begin{aligned}
S\left(y_{1}, y_{2}\right) & =\left(T_{1}\left(y_{1}, y_{2}\right), T_{2}\left(y_{1}, y_{2}\right)\right) \\
& =\left(Q_{1}\left(y_{1}, y_{2}\right)+Q_{2}\left(y_{1}, y_{2}\right), P_{1}\left(y_{1}, y_{2}\right)+P_{2}\left(y_{1}, y_{2}\right)\right) \\
& =\left(Q_{1}\left(y_{1}, y_{2}\right), P_{1}\left(y_{1}, y_{2}\right)\right)+\left(Q_{2}\left(y_{1}, y_{2}\right), P_{2}\left(y_{1}, y_{2}\right)\right) \\
& =A\left(y_{1}, y_{2}\right)+B\left(y_{1}, y_{2}\right) .
\end{aligned}
$$

In view of the proof above, we get the following. 
(I) For all $\left(y_{1}, y_{2}\right),\left(u_{1}, u_{2}\right) \in \Omega_{r}$, we find that

$$
\left\|A\left(y_{1}, y_{2}\right)+B\left(u_{1}, u_{2}\right)\right\|=\left\|Q_{1}\left(y_{1}, y_{2}\right)+Q_{2}\left(u_{1}, u_{2}\right)\right\|+\left\|P_{1}\left(y_{1}, y_{2}\right)+P_{2}\left(u_{1}, u_{2}\right)\right\| \leq r .
$$

Thus, $A\left(y_{1}, y_{2}\right)+B\left(u_{1}, u_{2}\right) \in \Omega_{r}$ for all $\left(y_{1}, y_{2}\right),\left(u_{1}, u_{2}\right) \in \Omega_{r}$.

(II) For $\left(y_{1}, y_{2}\right),\left(u_{1}, u_{2}\right) \in X, t \in[0,1]$,

$$
\begin{aligned}
\left\|B\left(y_{1}, y_{2}\right)-B\left(u_{1}, u_{2}\right)\right\| & =\left\|Q_{2}\left(y_{1}, y_{2}\right)-Q_{2}\left(u_{1}, u_{2}\right)\right\|+\left\|P_{2}\left(y_{1}, y_{2}\right)-P_{2}\left(u_{1}, u_{2}\right)\right\| \\
& \leq \frac{1}{2}\left\|\left(y_{1}-u_{1}, y_{2}-u_{2}\right)\right\|=\frac{1}{2}\left\|\left(y_{1}, y_{2}\right)-\left(u_{1}, u_{2}\right)\right\| .
\end{aligned}
$$

So, $B$ is a contraction mapping.

(III) The continuity of $Q_{1}$ and $P_{1}$ implies that the operator $A$ is continuous. Also, $A$ is uniformly bounded on $\Omega_{r}$ as

$$
\left\|A\left(y_{1}, y_{2}\right)\right\|=\left\|Q_{1}\left(y_{1}, y_{2}\right)\right\|+\left\|P_{1}\left(y_{1}, y_{2}\right)\right\| \leq 4 r .
$$

Moreover, for any $t_{1}, t_{2} \in[0,1]$,

$$
\begin{aligned}
& \left|A\left(y_{1}, y_{2}\right)\left(t_{2}\right)-A\left(y_{1}, y_{2}\right)\left(t_{1}\right)\right| \\
& \quad=\left|\left(Q_{1}\left(y_{1}, y_{2}\right)\left(t_{2}\right)-Q_{1}\left(y_{1}, y_{2}\right)\left(t_{1}\right), P_{1}\left(y_{1}, y_{2}\right)\left(t_{2}\right)-P_{1}\left(y_{1}, y_{2}\right)\left(t_{1}\right)\right)\right| \\
& \quad \leq\left|Q_{1}\left(y_{1}, y_{2}\right)\left(t_{2}\right)-Q_{1}\left(y_{1}, y_{2}\right)\left(t_{1}\right)\right|+\left|P_{1}\left(y_{1}, y_{2}\right)\left(t_{2}\right)-P_{1}\left(y_{1}, y_{2}\right)\left(t_{1}\right)\right| \\
& \quad \leq \lambda_{1} \frac{M_{1}}{\Gamma\left(v_{1}+1\right)}\left|t_{2}^{\nu_{1}}-t_{1}^{\nu_{1}}\right|+\lambda_{2} \frac{M_{1}}{\Gamma\left(v_{2}+1\right)}\left|t_{2}^{\nu_{2}}-t_{1}^{\nu_{2}}\right|,
\end{aligned}
$$

which is independent of $\left(y_{1}, y_{2}\right)$. Therefore, $A$ is equicontinuous on $\Omega_{r}$. Hence, by the Arzela-Ascoli theorem, $A$ is compact on $\Omega_{r}$.

Thus all the assumptions of Lemma 3.2 are satisfied, so $S\left(y_{1}, y_{2}\right)=A\left(y_{1}, y_{2}\right)+B\left(y_{1}, y_{2}\right)$ has at least one fixed point. Hence, we obtain that (1)-(4) has at least one solution.

\section{Conclusions}

There are few works that deal with multi-point boundary value problems for a coupled system of nonlinear fractional differential equations. In this article, we study multi-point boundary value problems for a coupled system of nonlinear fractional differential equations (1)-(4). By using Green's function, the Banach contraction principle and Krasnoselskii's fixed point theorem, we establish some new existence, uniqueness theorems of solutions for multi-point boundary value problems for a coupled system of nonlinear fractional differential equations (1)-(4).

Competing interests

The authors declare that they have no competing interests.

Authors' contributions

The authors declare that the study was realized in collaboration with the same responsibility. All authors read and approved the final manuscript.

\section{Acknowledgements}

The research was supported by the Youth Science Foundation of China (11201272) and the Science Foundation of Shanxi Province (2010021002-1).

Received: 25 January 2015 Accepted: 27 April 2015 Published online: 08 May 2015 


\section{References}

1. Diethelm, K: The Analysis of Fractional Differential Equations. Lecture Notes in Math. (2010)

2. Kilbas, AA, Srivastava, HM, Trujillo, JJ: Theory and Applications of Fractional Differential Equations. North-Holland Mathematics Studies, vol. 204. Elsevier, Amsterdam (2006)

3. Miller, KS, Ross, B: An Introduction to the Fractional Calculus and Differential Equations. Wiley, New York (1993)

4. Podlubny, I: Fractional Differential Equations. Academic Press, San Diego (1999)

5. Tarasov, VE: Fractional Dynamics: Application of Fractional Calculus to Dynamics of Particles, Fields and Media. Springer, Berlin (2010)

6. Zhang, S: Existence of positive solution for some class of nonlinear fractional differential equations. J. Math. Anal. Appl. 278, 136-148 (2003)

7. Benchohra, M, Henderson, J, Ntouyas, SK, Ouahab, A: Existence results for fractional order functional differential equations with infinite delay. J. Math. Anal. Appl. 338, 1340-1350 (2008)

8. Ahmad, B, Nieto, JJ: Existence results for a coupled system of nonlinear fractional differential equations with three-point boundary conditions. Comput. Math. Appl. 58, 1838-1843 (2009)

9. Chang, YK, Nieto, JJ: Some new existence results for fractional differential inclusions with boundary conditions. Math. Comput. Model. 49, 605-609 (2009)

10. Agarwal, RP, Benchohra, M, Hamani, S: A survey on existence results for boundary value problems of nonlinear fractional differential equations and inclusions. Acta Appl. Math. 109, 973-1033 (2010)

11. Zhou, Y, Jiao, F: Nonlocal Cauchy problem for fractional evolution equations. Nonlinear Anal., Real World Appl. 11 , 4465-4475 (2010)

12. Wang, J, Zhou, Y: A class of fractional evolution equations and optimal controls. Nonlinear Anal., Real World Appl. 12, 262-272 (2011)

13. Wang, G, Zhang, L, Song, G: Systems of first order impulsive functional differential equations with deviating arguments and nonlinear boundary conditions. Nonlinear Anal. 74, 974-982 (2011)

14. Sun, SR, Li, QP, Li, Y: Existence and uniqueness of solutions for a coupled system of multi-term nonlinear fractional differential equations. Comput. Math. Appl. 64, 3310-3320 (2012)

15. Mophou, GM: Existence and uniqueness of mild solutions to impulsive fractional differential equations. Nonlinear Anal. 72, 1604-1615 (2010)

16. Benchohra, M, Slimani, BA: Existence and uniqueness of solutions to impulsive fractional differential equations. Electron. J. Differ. Equ. 2009, 10 (2009)

17. Ahmad, B, Alsaedi, A: Existence and uniqueness of solutions for coupled systems of higher-order nonlinear fractional differential equations. Fixed Point Theory Appl. 2010, Article ID 364560 (2010)

18. Zhai, CB, Hao, MR: Fixed point theorems for mixed monotone operators with perturbation and applications to fractional differential equation boundary value problems. Nonlinear Anal. 75, 2542-2551 (2012)

19. Yang, C, Zhai, CB: Uniqueness of positive solutions for a fractional differential equation via a fixed point theorem of a sum operator. Electron. J. Differ. Equ. 2012, 70 (2012)

20. Zhai, CB, Yan, WP, Yang, C: A sum operator method for the existence and uniqueness of positive solutions to Riemann-Liouville fractional differential equation boundary value problems. Commun. Nonlinear Sci. Numer. Simul. $18,858-866(2013)$

21. Su, X: Boundary value problem for a coupled system of nonlinear fractional differential equations. Appl. Math. Lett. 22, 64-69 (2009)

22. Bai, CZ, Fang, JX: The existence of a positive solution for a singular coupled system of a nonlinear fractional differential equations. Appl. Math. Comput. 150(3), 611-621 (2004)

23. Goodrich, CS: Existence of a positive solution to systems of differential equations of fractional order. Comput. Math. Appl. 62, 1251-1268 (2011)

24. Dunninger, D, Wang, H: Existence and multiplicity of positive solutions for elliptic systems. Nonlinear Anal. 29, 1051-1060 (1997)

25. Krasnoselskii, MA: Topological Methods in the Theory of Nonlinear Integral Equations. Pergamon, New York (1964)

\section{Submit your manuscript to a SpringerOpen ${ }^{\circ}$ journal and benefit from:}

- Convenient online submission

Rigorous peer review

- Immediate publication on acceptance

- Open access: articles freely available online

- High visibility within the field

- Retaining the copyright to your article 